\title{
The Neuronal MicroRNA miR-326 Acts in a Feedback Loop with Notch and Has Therapeutic Potential against Brain Tumors
}

\author{
Benjamin Kefas, ${ }^{1}$ Laurey Comeau, ${ }^{1}$ Desiree H. Floyd, ${ }^{1}$ Oleksandr Seleverstov, ${ }^{1}$ Jakub Godlewski, ${ }^{2}$ Tom Schmittgen, ${ }^{3}$ \\ Jinmai Jiang, ${ }^{3}$ Charles G. diPierro, ${ }^{4}$ Yunqing Li, ${ }^{5}$ E. Antonio Chiocca, ${ }^{2}$ Jeongwu Lee, ${ }^{6}$ Howard Fine, ${ }^{6}$ Roger Abounader, ${ }^{1}$ \\ Sean Lawler, ${ }^{2}$ and Benjamin Purow ${ }^{1}$ \\ ${ }^{1}$ Division of Neuro-Oncology, Neurology Department, University of Virginia Health System, Charlottesville, Virginia 22908, ${ }^{2}$ Dardinger Laboratory for \\ Neuro-Oncology and Neurosciences, Department of Neurological Surgery, Ohio State University Medical Center, Columbus, Ohio 43210-1240, ${ }^{3}$ College of \\ Pharmacy, Ohio State University, Columbus, Ohio 43210-1291, ${ }^{4}$ Pathology Department, University of VIrginia Health System, Charlottesville, Virginia \\ 22908-0816, ${ }^{5}$ Neurology Department, The Johns Hopkins University School of Medicine and The Hugo W. Moser Research Institute at Kennedy Krieger, \\ Baltimore, Maryland 21205-1832, and 'Neuro-Oncology Branch, National Cancer Institute/National Institute of Neurological Disorders and Stroke, \\ National Institutes of Health, Bethesda, Maryland 20892
}

Little is known of microRNA interactions with cellular pathways. Few reports have associated microRNAs with the Notch pathway, which plays key roles in nervous system development and in brain tumors. We previously implicated the Notch pathway in gliomas, the most common and aggressive brain tumors. While investigating Notch mediators, we noted microRNA-326 was upregulated following Notch-1 knockdown. This neuronally expressed microRNA was not only suppressed by Notch but also inhibited Notch proteins and activity, indicating a feedback loop. MicroRNA-326 was downregulated in gliomas via decreased expression of its host gene. Transfection of microRNA-326 into both established and stem cell-like glioma lines was cytotoxic, and rescue was obtained with Notch restoration. Furthermore, miR-326 transfection reduced glioma cell tumorigenicity in vivo. Additionally, we found microRNA-326 partially mediated the toxic effects of Notch knockdown. This work demonstrates a microRNA-326/Notch axis, shedding light on the biology of Notch and suggesting microRNA-326 delivery as a therapy.

\section{Introduction}

MicroRNAs (miRNAs or miRs) are a class of small noncoding RNAs recently discovered to be endogenously expressed in plants and animals, including humans. A microRNA is first transcribed from an intron or intergenic region as a long primary microRNA (pri-miRNA), then processed in the nucleus to a pre-microRNA hairpin (pre-miRNA) by the ribonuclease enzyme Drosha. The pre-miRNA is exported to the cell cytoplasm, where it is excised by Dicer to a 19-23 nt mature form that binds with partial complementarity to sites in the $3^{\prime}$-UTR of numerous target genes (Murchison and Hannon, 2004). Binding of miRNAs leads to interference with translation or, less frequently, cleavage of target mRNAs (Yekta et al., 2004). miRNAs have been implicated in the regulation of stem cells, differentiation, and tumor formation and inhibition (Chan et al., 2005; Ciafrè et al., 2005; Croce and Calin, 2005). There is,

Received 0ct. 6, 2009; accepted 0ct. 13, 2009.

This work was supported by National Institutes of Health Grant R01CA136803 (B.P.) and by startup funding from the University of Virginia Neurology Department and Cancer Center (B.P.). We thank Joanne Lannigan and Michael Solga for assistance with flow cytometry. We are grateful to Spyros Artavanis-Tsakonas for his kind gift of the Notch-1 expression vector and to S. Diane Hayward for her kind gift of the CBF1-luciferase reporter plasmid.

Correspondence should be addressed to Benjamin Purow, University of Virginia Health System, Old Medical School—Room 4814, 21 Hospital Drive, Charlottesville, VA 22908. E-mail: bwp5g@virginia.edu.

DOI:10.1523/JNEUROSCI.4966-09.2009

Copyright $\odot 2009$ Society for Neuroscience $\quad$ 0270-6474/09/2915161-08\$15.00/0 however, little known of the regulation of miRNAs and their interaction with major signaling pathways.

We showed previously that microRNA-7 inhibits two central oncogenic pathways in cancer, epidermal growth factor receptor (EGFR) and the Akt pathway (Kefas et al., 2008). Both pathways play a major role in the pathogenesis of gliomas, the most common and lethal brain tumor in adults. We demonstrated in other work that the Notch pathway also plays an important role in glioma cell survival (Purow et al., 2005). Notch is critical in stem cell maintenance and cell survival, as well as in cell fate decisions such as neuronal versus glial fate in the developing nervous system (Henrique et al., 1997; Gaiano et al., 2000; Morrison et al., 2000; Amsen et al., 2004; Oishi et al., 2004; Weng et al., 2004; Stylianou et al., 2006). The four Notch receptors are activated via binding by a ligand on an adjacent cell. This triggers enzymatic cleavages by $\alpha$-secretase and $\gamma$-secretase that liberate the Notch intracellular domain (NICD), which travels to the nucleus and recruits activators to the $\mathrm{CBF} 1$ transcription factor. This drives expression of Notch mediators such as the Hes and Hey transcription factors, but clearly other mediators of Notch have yet to be discovered.

To find potential microRNA mediators of Notch effects in glioma, we performed miRNA microarray analysis of glioma tumor stem cells transfected with either control siRNA or Notch-1 siRNA. One of the miRNAs significantly increased 
A

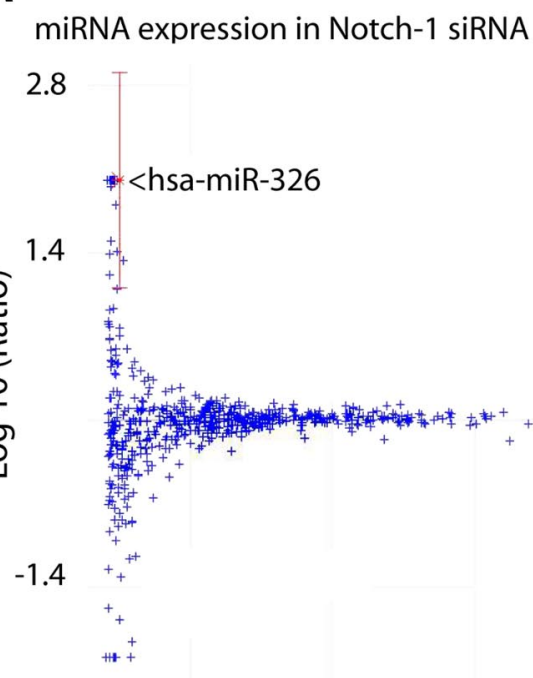

$-2.8$
B

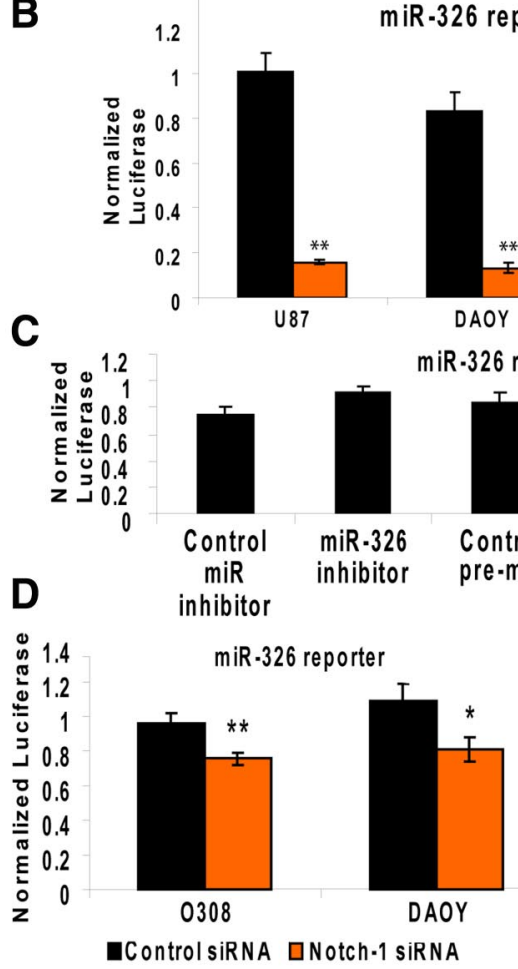

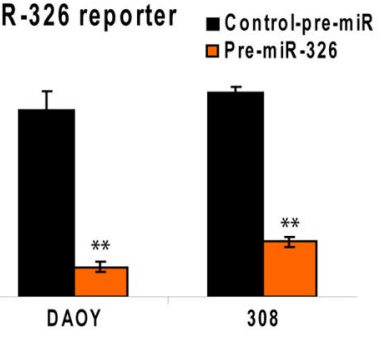

iR-326 reporter
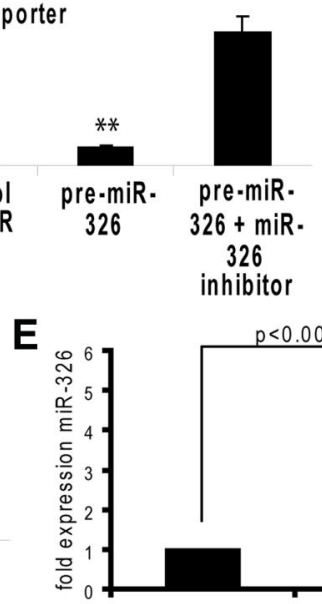

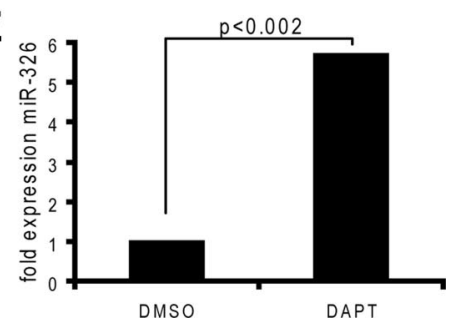

Figure 1. Notch-1 knockdown increases expression and activity of miR-326. A, Plot of microRNA microarray expression data from 0308 GTSC cells transfected with Notch-1 siRNA versus control siRNA, with log relative expression in Notch-1 siRNA samples on the $y$-axis and log intensity on the $x$-axis. Increased expression of miR-326 in Notch-1 siRNA samples versus control siRNA samples is shown $(p<0.05)$. Note that the analysis software automatically sets the fold change at 100 -fold when the signal in one of the comparators is undetectable. $\boldsymbol{B}$, Sensitivity of miR-326 reporter to miR-326 in brain tumor lines. Normalized luciferase activity of miR-326 activity reporter plasmid in U87MG and 0308 tumor stem cell glioma lines and DAOY medulloblastoma cell line $3 \mathrm{~d}$ after transfection with control pre-miR or pre-miR-326 $\left.{ }^{* *} p<0.01\right)$. C, miR-326 reporter is responsive to both miR-326 and a miR-326 inhibitor. Normalized luciferase activity of miR-326 activity reporter plasmid in DAOY cells $3 \mathrm{~d}$ after transfection with control pre-miR or pre-miR-326 alone or together with control miR inhibitor or miR-326 inhibitor $\left({ }^{* *} p<0.01\right)$. D, Notch-1 knockdown increases miR-326 activity. Normalized luciferase activity of miR-326 activity reporter plasmid in the $0308 \mathrm{GTSC}$ line or the DAOY medulloblastoma cell line $3 \mathrm{~d}$ after transfection with control siRNA or Notch-1 siRNA $\left({ }^{* *} p<0.01\right.$ and $\left.{ }^{*} p<0.05\right)$. E, A small-molecule Notch inhibitor increases miR-326 expression. Q-PCR indicating normalized miR-326 expression of $0308 \mathrm{GTSC}$ cells treated for $4 \mathrm{~d}$ with 25 $\mu$ M DAPT or equal vol:vol DMSO vehicle $(p<0.002)$.

with Notch-1 knockdown was miR-326, first identified in a report of microRNAs expressed in neurons (Kim et al., 2004). miR-326 had also been noted previously on a list of microRNAs elevated in zebrafish embryos treated with a Notch inhibitor (Thatcher et al., 2007). Our work not only indicated suppression of this microRNA by Notch, but also showed that Notch pathway members and activity were inhibited by miR-326. We found low expression of miR-326 in gliomas, and forced expression of this miRNA in glioma cells was cytotoxic in both standard glioma lines and more resistant glioma tumor stem cell-like lines. Transfection with miR-326 markedly reduced in vivo tumorigenicity of glioma cells in an orthotopic mouse model. Importantly, rescue experiments demonstrated that the phenotypic effects of Notch and miR-326 were each partially mediated by suppression of the other. These results suggest a microRNA-326/Notch axis with potential therapeutic implications.

\section{Materials and Methods}

Cell culture. Glioma cell lines U87MG, U251MG, T98G, U373MG, A172, and medulloblastoma cell line DAOY were all acquired from American Type Culture Collection. Tumor stem cell line 0308 was derived and validated as described previously (Lee et al., 2006). All cell lines were grown under previously described conditions (Purow et al., 2005; Kefas et al., 2008).

Materials. Pre-miR-control and pre-miR-326 were purchased from Applied Biosystems/Ambion; control-miR-hairpin-inhibitor and miR-326-hairpin-inhibitor were purchased from Thermo Scientific
Dharmacon. Notch-2 siRNA was from Santa Cruz Biotechnology, Notch-1 control siRNAs was from Qiagen (Purow et al., 2005), and control siRNAs were from Santa Cruz Biotechnology and Qiagen. The $\gamma$-secretase inhibitor DAPT ( $N$-[(3,5-difluorophenyl)acetyl $]$-Lalanyl-2-phenyl]glycine-1,1-dimethylethyl ester) was obtained from Sigma-Aldrich.

Transfection of cells. Notch-1 siRNA and microRNA-326 transfections were as described previously using the oligofectamine reagent (Invitrogen) at $20 \mathrm{~nm}$ (Purow et al., 2005). For 0308 tumor stem cells, the plates were first coated with laminin and poly-L-ornithine (Sigma-Aldrich). Transfection of reporter plasmids and the Notch-1 full-length plasmid were done with Fugene HD or Fugene 6 for U87 (Roche Diagnostics). Plasmid transfections were in six-well plates with $1.0 \mu \mathrm{g}$ of reporter plasmid plus $0.05 \mu \mathrm{g}$ of CMV- $\beta$-galactosidase or with $1.5 \mu \mathrm{g}$ of Notch$1 /$ control plasmid.

MicroRNA microarray. The 0308 GBM tumor stem cell line was transfected with Notch-1 or control siRNAs as described and frozen pelleted cells shipped to Cogenics (a division of Clinical Data). RNA was extracted from frozen cell pellets using the Qiagen miRNeasy Mini Kit, essentially as described by the manufacturer (Qiagen Part \#217004). RNA samples (100 ng) were labeled using the miRNA Complete Labeling and Hyb Kit from Agilent Technologies (Part \#5190-0408) as described by the manufacturer. Labeled cRNAs were hybridized to Agilent Human (V2) miRNA Microarrays (part \# G4470B) and scanned with an Agilent DNA Microarray Scanner. Scanned image files were visually inspected and converted into data files using Agilent Feature Extraction Software. Two replicates were performed with each experimental condition, and two arrays were performed for each of those replicates. The miRNA ex- 
A
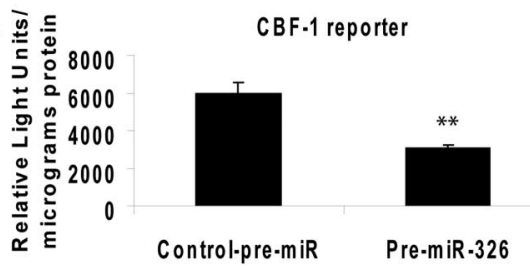

B

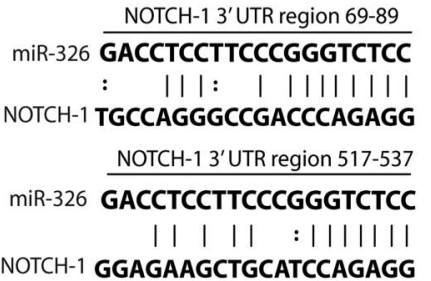

C
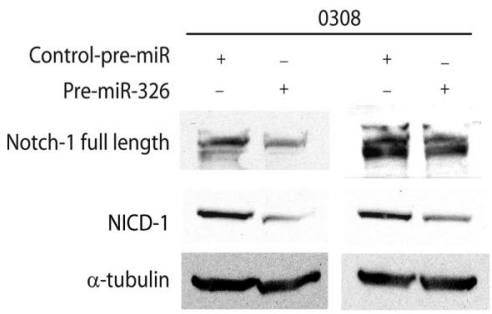

D

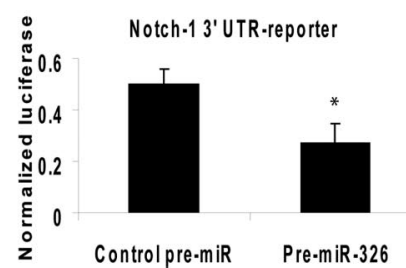

Figure 2. miR-326 inhibits the Notch pathway. A, miR-326 transfection decreases activity of a Notch reporter plasmid. Luciferase activity normalized with protein concentration of $\mathrm{U} 251 \mathrm{MG}$ cells stably expressing (BF-1 reporter plasmid $3 \mathrm{~d}$ after transfection with control pre-miR or pre-miR-326 ${ }^{* *} p<0.01$ ). miR-326 targets Notch-1. B, Sites of miR-326 seed matches in the Notch-1 $3^{\prime}$-UTR. C, Immunoblot showing protein expression of full-length and intracellular domain of Notch-1 (NICD-1) in the 0308 tumor stem cell line $3 \mathrm{~d}$ after transfection with control pre-miR or pre-miR-326 ( $\alpha$-tubulin loading controls shown at bottom). D, Normalized activity of Notch-1 $3^{\prime}$-UTR luciferase reporter plasmid $3 \mathrm{~d}$ after transfection with control pre-miR or pre-miR-326 $\left({ }^{*} p<0.05\right)$. miR-326 targets Notch-2. $\boldsymbol{E}$, Sites of miR-326 seed matches in the Notch-2 $3^{\prime}$-UTR. $\boldsymbol{F}$, Immunoblot showing protein expression of Notch-2 in U251MG cells $3 \mathrm{~d}$ after transfection with control pre-miR or pre-miR-326 ( $\alpha$-tubulin loading controls shown at bottom). G, Normalized activity of Notch-23'-UTR luciferase reporter plasmid $3 \mathrm{~d}$ after transfection with control pre-miR or pre-miR-326 $\left({ }^{*} p<0.05\right)$.

pression data from the biologic replicates were combined into an error-weighted average using the Rosetta Resolver Gene Expression Data Analysis System. Comparisons were made for expression of each microRNA between the Notch-1 siRNA and control siRNA conditions, generating metrics including fold change and $p$ value.

Plasmid construction. For the Notch-1 and -2 3'-UTR reporter plasmids, these regions were first amplified from human genomic DNA using High-Fidelity polymerase enzyme (New England Biolabs) and inserted into the unique XbaI restriction site $3^{\prime}$ to the luciferase gene in the pGL3promoter plasmid (Promega). miR-326 reporter insert (three copies of the full site complementary to miR-326) were placed into the multiple cloning site of p-miR-luciferase vector using HindIII and SpeI restriction enzymes (Applied Biosystems/Ambion.

Primer sequences were as follows: Notch-1-3'-UTR-F, 5' -GTAACTTCTAGACGACCAGAGGAGCCTTTTTAAAAC-3'. Notch-1-3'-UTR-R, 5'-GTACCATCTAGACACCATCAGTATCATTTTTATTGC-3'. Notch-23'-UTR-F, 5'-GTAACTGCTAGCCTCCAGTGTAGAGACATAACTG-3'. Notch-2-3'-UTR-R, 5'-GTACCAAAGCTTCATGTTCAAATATCTCACTGAC-3'.

Real-time PCR for Figure 3 studies. Patient samples were obtained with approval from the Ohio State University and University of Virginia Institutional Review Boards. Total RNA from patient samples was extracted according to the standard Trizol protocol (Invitrogen). For endogenous controls, cDNA was synthesized using random hexamers with the iScript cDNA Synthesis Kit (Bio-Rad) and $1 \mu \mathrm{g}$ of total RNA. For mature microRNA expression analysis, cDNA was synthesized using the TaqMan MicroRNA Reverse Transcription Kit (Applied Biosystems) and 10 ng of total RNA along with miR-326-specific primer supplied with the miR-326 Taqman MicroRNA Assay (Applied Biosystems). Quantitative real-time PCR analysis was performed using the 7500 Real Time PCR System (Applied Biosystems). A human 18S rRNA TaqMan probe (Applied Biosystems) was used as endogenous control (Jiang et al., 2005). For $\beta$-arrestin real-time PCR, the following primers were used: $\beta$-arrestin $1-\mathrm{F}, 5^{\prime}$ GACCACCAGGCAGTTCCTC- $3^{\prime} \quad \beta$-arrestin 1-R, 5'-TGGACGTTGACGCTGATG-3'.

Real-time PCR for Figure 1E studies. RNA was isolated using QIAshredder and RNeasy columns (Qiagen). RNA was treated with DNase I (Invitrogen). RT-PCR of $1 \mu \mathrm{g}$ of RNA was performed using the miScript Reverse Transcription Kit (Qiagen) and a miR-326specific Primer Assay ( $5 \mu$ l per reaction) and Universal Primers (5 $\mu$ l per reaction) (both from Qiagen). $\alpha$-Tubulin expression was used as a control, with primer sequences AGATCATTGACCTCGTGTTGGA and ACCAGTTCCCCCACCAAAG. The Bio-Rad iCycler was used to carry out quantitative PCR using a hot start, with 56 annealing (45 s) and 72 extension ( $45 \mathrm{~s}$ ) for 40 cycles, followed by a melt curve analysis.

Immunoblots. Immunoblots were performed as previously described (Purow et al., 2005). Primary antibodies included anti-Notch-1 (mN1A) (Santa Cruz Biotechnology), antiNotch-2 (8A1), anti-PARP, and anti- $\alpha$ tubulin (11H10) (Cell Signaling Technology). Horseradish peroxidase-conjugated secondary antibodies to rabbit or mouse IgG were used (1:10,000; Jackson ImmunoResearch Laboratories).

Flow cytometry analysis of apoptosis/ propidium iodide and Hoescht 33342 staining. Propidium iodide (PI) cell cycle flow cytometry was performed on a FACSVantage SE (Becton Dickinson), and propidium iodide (PI)/Hoescht 33342 (HO) (Sigma) fluorescence microscopy (Axiovert 135M, Carl Zeiss) assays were performed as previously described (Kefas et al., 2003, 2008; Purow et al., 2005).

Caspase- $3 / 7$ assay. Cells transfected with either control pre-miR or miR326 were washed with $1 \times$ PBS once and resuspended in $100 \mu$ lof PBS. Forty microliters of the cells were mixed with $40 \mu \mathrm{l}$ of caspase-Glo 3/7 reagent (Promega) and incubated for $1 \mathrm{~h}$ at room temperature with agitation. Luciferase activity was measured as previously described (Purow et al., 2005).

Luciferase assay. Luciferase reporter assays were performed as previously described on a Promega Glomax 20/20 luminometer (Purow et al., 2005). CBF1, Notch-1 3'-UTR, and Notch-2 3 '-UTR-luciferase activities were double normalized by dividing each well by both $\beta$-galactosidase activity and the average luciferase/ $\beta$-galactosidase value in a parallel set done with constitutive luciferase plasmid. To measure downstream Notch activity, we generated 0308 brain tumor stem cells stably expressing a luciferase reporter driven by RBP-Jk (CSL/CBF1/Su (H)/Lag1) (SABiosciences), a transcription factor and mediator of canonical Notch signaling. The RBP-Jk-responsive luciferase vector encodes the firefly luciferase gene under the control of a minimal $(\mathrm{m}) \mathrm{CMV}$ promoter and tandem repeats of the RBP-Jk transcriptional response element. To generate the stable cell line, 0308 cells were infected with the lentivirus bear- 
ing RBP-Jk-responsive element with luciferase. The infected cells were then selected using puromycin antibiotic (Sigma-Aldrich). The resultant stable line was used in several assays with siRNAs transfection. 0308/CBF1-luciferase cells were plated at a density of $10^{5}$ per well in a six-well plate overnight and transfected with four different control or Notch-1, $-2,-3$, or -4 siRNAs, and luciferase activity (luminescence) was measured $3 \mathrm{~d}$ following transfection. Luminescence was assessed with the luciferase assay system (Promega) per the manufacturer's protocol on an EG\&G Berthold Lumat LB9507. To normalize luciferase activity, absolute luminescence for each sample was divided by the protein concentration for that sample. Protein concentration was determined by the DC Protein Assay kit II (Bio-Rad Life Science) per the manufacturer's instructions.

Soft agar colony-forming assay. A $0.6 \%$ agar/ medium base layer was made, and $3 \mathrm{ml}$ was added to each well (six-well plate) to prevent cells from attaching and forming a monolayer on the plastic substrate. One milliliter of culture medium containing cells transfected with either control pre-miR or pre-miR-326 was mixed with $1 \mathrm{ml}$ of $0.6 \%$ agar/medium, poured on the base layer and allowed to solidify, and immediately placed in an incubator at $37^{\circ} \mathrm{C}$ and $5 \% \mathrm{CO}_{2}$. Medium was changed every 2-3 d, and after $21 \mathrm{~d}$, cells were stained with Wright's stain (Sigma-Aldrich) and counted under the microscope.

Tumor formation in vivo. U87 cells were transfected with control premiR or pre-miR-326 for $48 \mathrm{~h}$. The transfected cells were then counted and $2 \times 10^{5}$ were stereotactically (Stoelting) implanted into the right corpus striatum of immunocompromised SCID/NCr BALB/c adult male mice ( $n=7$ for each condition). Cerebral magnetic resonance imaging was performed on anesthetized mice at 2 weeks. Ten to fifteen minutes before scanning, $30 \mu \mathrm{l}$ of Magnevist brand gadopentetate dimeglumine was injected intraperitoneally. T1-weighted serial coronal images of each brain were acquired at $1 \mathrm{~mm}$ intervals with a $5 \times 5 \mathrm{~mm}$ field and a $256 \times$ 256 pixel resolution. For image analysis and tumor volume quantification, a luminosity histogram was first generated for a selected area of the left cerebrum that was grossly tumor-free. This served as an internal control. Pixel luminosity mean and SD were noted. Histogram generation was repeated on a similar selection from the right cerebrum that contained all enhancing tumor. Pixels in the right cerebrum greater than two SDs above the left cerebrum control luminosity mean were recorded as representing enhanced tumor for a given image. This procedure was then repeated for all the images showing enhanced tumor for a given brain, thus generating a sum of enhanced tumor pixels for each brain. Tumor volume relates to tumor pixels in a linear manner and was calculated based on the image acquisition interval distance and resolution (Amos et al., 2007).

\section{Results}

\section{Notch inhibition increases the expression and activity} of miR-326

We transfected glioma tumor stem cells with either control siRNA or with an efficient Notch-1 siRNA that we validated previously (Purow et al., 2005), followed by miRNA expression profiling by microarray. miR-326 was one of the miRNAs that was significantly increased (Fig. 1A) $(p<0.05)$. To further test whether miR-326 expression or activity increases with Notch-1 knockdown, we functionally examined miR-326 activity with a luciferase reporter plasmid that included miR-326 target sites. To validate the reporter, we tested the effects of transfection with pre-miR-326 and miR-326 inhibitor. miR-326 lowered luciferase activity of the reporter, while miR-326 inhibitor blocked this effect in cells cotransfected with both control miRNA and miR326 inhibitor (Fig. 1B,C). Cells transfected with Notch-1 siRNA demonstrated significantly increased miR-326 activity, as reflected in decreased miR-326 reporter output (Fig. 1D). This lowering of miR-326 reporter was abolished when miR-326 inhibitor was introduced (data not shown). The increase in miR326 expression following Notch inhibition was confirmed by real-time PCR in 0308 GTSC cells treated with DAPT, a well established $\gamma$-secretase inhibitor that blocks Notch activity, $2.6 \pm$ 0.57 - versus $6.46 \pm 0.64$-fold (Fig. $1 E)(p<0.002)$. These data indicate that miR-326 is regulated by Notch activity.

\section{miR-326 inhibits the Notch pathway}

We noted potential target sites (seed matches) for miR-326 in both the Notch-1 and Notch-2 3 '-UTRs. We therefore tested the effects of pre-miR-326 transfection on Notch activity, using a glioma line we reported previously that stably expresses a well established CBF-1 luciferase reporter (Hsieh et al., 1997; Purow et al., 2005). miR-326 lowered Notch activity by $>50 \%, 5927.7 \pm$ 629 versus $3049 \pm 156$ (Fig. $2 A)(p<0.002)$. We then assessed the targeting of Notch- 1 and Notch- 2 by miR-326, given the seed matches noted in both $3^{\prime}$-UTRs (Fig. 2 B,E). Pre-miR-326 transfection caused substantial decreases in both Notch-1 and Notch-2 protein by immunoblot (Fig. $2 C, F$ ). In addition, it significantly reduced output from Notch-1 and Notch-2 luciferase/ $3^{\prime}$-UTR reporter plasmids, $0.5 \pm 0.06$ versus $0.7 \pm 0.08$ and $0.83 \pm$ 0.05 versus $0.59 \pm 0.03$, respectively (Fig. $2 D, G)(p<0.05)$. These effects of miR-326 appear specific, as they were not observed when a miR-7 reporter plasmid was used (data not shown).

\section{miR-326 and its host gene $\beta$-arrestin 1 are downregulated in glioblastoma}

Since several miRNAs have been identified as dysregulated in gliomas (Chan et al., 2005; Ciafrè et al., 2005; Kefas et al., 2008), we set out to measure the levels of mature miR-326 in GBM tissues versus surrounding brain using Q-PCR. miR-326 levels were indeed significantly decreased in a set of five GBMs, $1 \pm 0.0$ versus $0.18 \pm 0.0002$-fold (Fig. $3 A)(p<0.001)$. Similar results 
A

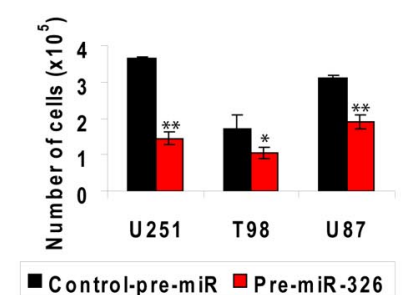

C
B
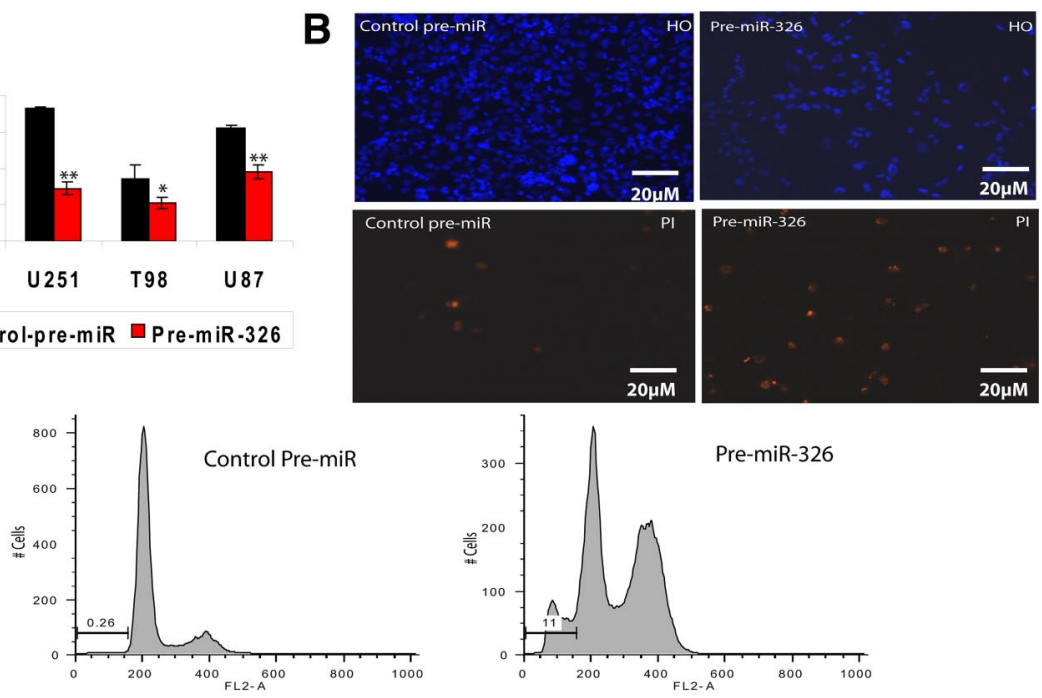

D
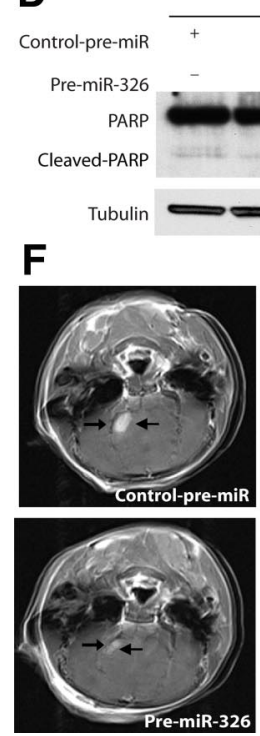

T98
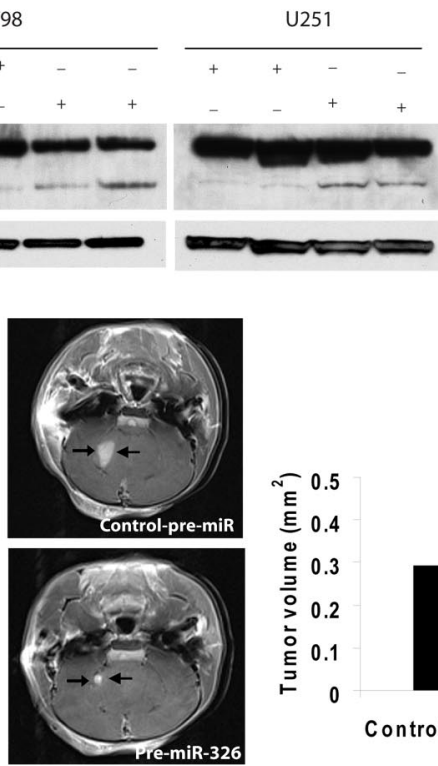

E

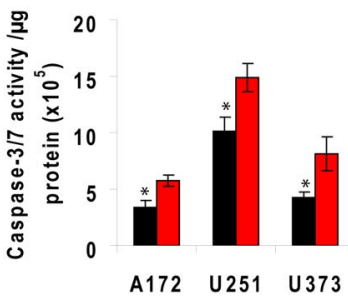

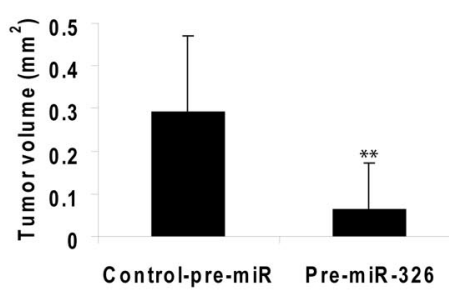

Figure 4. miR-326 decreases cell viability of established glioma cell lines. A, Transfection with miR-326 decreases cell counts of glioma cells. Plot of cell counts by microscopy with a hemocytometer performed $5-6 \mathrm{~d}$ after transfection with control pre-miR or miR-326 in U251MG, U87MG, and T98MG established glioma lines ( ${ }^{*} p<0.05$ and ${ }^{* *} p<0.01$ respectively). $\boldsymbol{B}$, Nuclear permeability assay demonstrates cell death in glioma cells following miR-326 transfection. Photomicrograph of U251MG glioma cells stained with propidium iodide (lower photos) and Hoechst (upper photos) $6 \mathrm{~d}$ after transfection with control pre-miR or miR-326. C, Cell cycle assay indicates apoptosis in miR-326-transfected glioma cells. Propidium iodide cell cycle and DNA fragmentation assays of U373MG cells by FACS $6 \mathrm{~d}$ after transfection with control pre-miR or pre-miR-326. Cleaved PARP and caspase 3/7 assays further support apoptosis in glioma cells transfected with miR-326. D, Immunoblots of full-length and cleaved PARP protein in T98G and U251MG glioma cell lines $6 \mathrm{~d}$ after transfection with control pre-miR or pre-miR-326. Bottom, $\alpha$-Tubulin as loading control. $\boldsymbol{E}$, Plot of caspase-3/7 activity normalized with protein concentration in A172, U251MG, and U373MG glioma cell lines $4 \mathrm{~d}$ after transfection with control pre-miR or pre-miR-326 $\left({ }^{*} p<0.05\right)$. $\boldsymbol{F}$, Effect of pre-miR-326 or control pre-miR on in vivo U87 glioma xenograft growth in the brains of immunodeficient mice. Representative brain MRI images showing smaller tumors in two mice from the miR-326 group versus two mice from the control group are shown. Mean tumor sizes in the two groups are also plotted and show a significant difference $\left({ }^{* *} p<0.01\right)$, with tumor sizes calculated as described in Materials and Methods.
- Control-pre-miR $\square$ Pre-miR-326

with miR-326 levels in individual samples (Fig. 3C,D). This indicated that miR-326 expression is decreased in GBM through decreased transcription of its host gene.

\section{miR-326 decreases glioma cell} proliferation and induces apoptosis

To determine the effect of miR-326 on cellular proliferation, cell counts were performed. miR-326 transfection decreased glioma cell numbers (Fig. $4 A$ ) in multiple glioma lines. To further investigate the role of miR-326 in brain tumors, established glioma cell lines were transfected with either control pre-miR or pre-miR326. After the addition of PI or HO, the number of cells that had taken up these dyes was imaged and quantified by fluorescence microscopy (Fig. $4 B$ ). While all cell nuclei stained for HO, only cells transduced with miR-326 had high levels of nuclear PI staining. Furthermore, numerous miR-326-transduced cells detached from the culture dish into the medium, resulting in fewer attached Hoechst-positive cells (Fig. $4 \mathrm{~B}$ ). Cell cycle assay indicated high numbers of sub- $\mathrm{G}_{0} / \mathrm{G}_{1}$ cells, indicating apoptosis (Fig. 4C). In addition to DNA fragmentation, caspase activation and poly(ADP-ribose) polymerase (PARP) cleavage are other hallmarks of apoptosis (Los et al., 2002). To demonstrate both phenomena following miR-326 transfection, we measured caspase- $3 / 7$ activity using a luminometry assay and assessed PARP cleavage using immunoblot. There was a significant increase in caspase-3/7 activity and elevated PARP cleavage in miR-326-transfected cells compared with control (Fig. $4 D, E$ ). To determine whether the in vitro effects of miR-326 translated to reduced tumor growth in vivo, U87 MG glioma cells were stereotactically implanted into the brains of immunocompromised mice after in vitro transfection with either control pre-miR or pre-miR-326. All animals $(n=7)$ implanted with control-premiR U87 MG cells developed substantial tumors $\left(0.3 \pm 0.18 \mathrm{~mm}^{3}\right)$, compared with no tumors in five of seven animals implanted with miR-326-transfected U87 MG cells and small tumors in the remaining two mice $\left(0.06 \pm 0.11 \mathrm{~mm}^{3}\right)$. This translated to were observed in another set of 11 GBM samples $(0.03 \pm 0.004)$ when compared with six normal brain samples $(0.2 \pm 0.09)$ (Fig. $3 B)(p<0.001)$. We reasoned that the downregulation of miR326 could be due either to decreased expression of its host gene or to decreased processing. To distinguish between these possibilities, we quantified the levels of the host gene for miR-326, $\beta$-arrestin 1, using RT-PCR of the second set of tissue samples. $\beta$-arrestin 1 levels were markedly decreased in GBMs (0.095 \pm $0.01)$ compared with normal brain tissues $(0.4 \pm 0.1)$ (Fig. $3 C$ ) $(p<0.001)$, and the levels of $\beta$-arrestin 1 correlated strikingly a significant difference in mean tumor size (Fig. $4 F)(p<0.01)$.

miR-326 decreases viability, proliferation, and invasiveness of glioblastoma stem cell-like lines

In our previous report comparing gene expression in validated glioblastoma-derived tumor stem cells (GTSCs) with that in GBM cells grown in standard conditions, microarray data indicated higher expression of Notch-1 and its ligands in GTSCs, possibly indicating an important role for Notch activity in these cells [supplemental material in (Lee et al., 2006)]. To assess the 
importance of different Notch family members in the viability of GTSCs in vitro, we transfected three GTSC lines with Notch-1, -2, -3, and -4 siRNAs. Interestingly, knockdown of each of the four Notch proteins decreased cell numbers in the GTSC lines assessed (Fig. 5A). The effects of the different Notch family siRNAs on the growth of GTSC were repeated when GTSC 0308 cells were transfected with four different control siRNAs, indicating that this was not a nonspecific effect of the control siRNA (Fig. 5B). To confirm the individual effects of the Notch-1 to -4 siRNAs used in this work, we tested their effect on Notch activity. 0308 glioma cells stably expressing a Notch reporter showed reduced luciferase activity from each of the Notch-1 to -4 siRNAs versus several control siRNAs (Fig. $5 C$ ). These data suggest that Notch family members play nonredundant roles in GTSC growth.

Since miR-326 decreased protein levels of Notch-1 and -2, we predicted that this miRNA would be cytotoxic to GTSCs. Cell cycle assay of GTSCs transfected with miR-326 by fluorescence-activated cell sorting (FACS) indeed demonstrated apoptosis, as shown by an increase in cells with sub- $\mathrm{G}_{0} / \mathrm{G}_{1}$ nuclei (Fig. $5 D$ ). miR-326 transfection also caused an increase in PARP cleavage and caspase-3/7 activation $(2.1 \pm 0.26$ vs $4.0 \pm 0.9, p<0.05)$, as well as a decrease in cell counts (Fig. $5 E, F$ ). Since invasion and colony formation are markers of a cancer's malignant potential in vivo, we assayed the impact of miR-326 on these traits in GTSCs. Transfection with pre-miR-326 decreased invasion through collagen IV-coated transwells by GTSCs (73.3 \pm 37 vs $10.6 \pm 8.1$ ) (Fig. $5 H$ ) $(p<0.05)$, and it also lowered clonogenicity in soft agar (Fig. 5I) $(p<0.01)$.

\section{Notch and miR-326 effects are each partially mediated by suppression of the other}

miR-326 has numerous predicted targets in addition to those in the Notch pathway, and some of them could conceivably be involved in the cytotoxic effects of miR326. To assess whether Notch inhibition plays a role in miR-326 activity, we determined whether a Notch expression plasmid lacking $3^{\prime}$-UTR could rescue a glioma line from the miR-326-driven decrease in cell viability. Following transfection of cells with either Notch-1 or control plasmid and pre-miR-326 or control pre-miR, we noted a large degree of rescue (Fig. 6A). We also tested whether miR-326 suppression by Notch played a role in the phenotypic effects of Notch inhibition. Given our previous result showing the effectiveness of a miR-326 inhibitor (Fig. 1), we tested whether it could influence the effects of efficient Notch-1 knock-

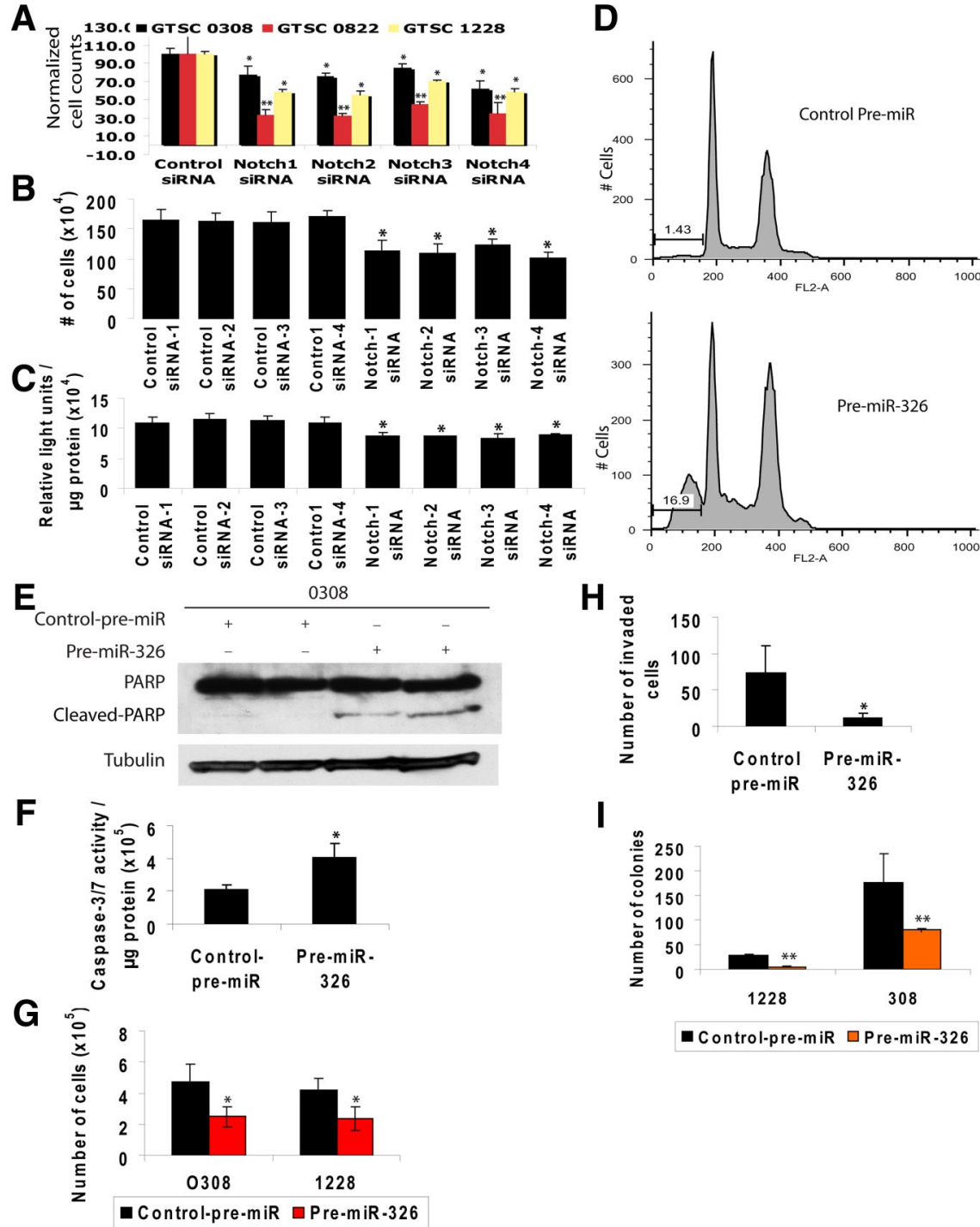

Figure 5. miR-326 decreases viability and invasiveness of glioblastoma stem cells., Decreased viability of GTSC lines transfected with siRNAs to Notch family members. $A, B$, Plots of cell counts of 0822,1228 , and 0308 glioma stem cells (normalized to $100 \%$ for control siRNA) $(\boldsymbol{A})$ and 0308 glioma stem cells $(\boldsymbol{B}) 5 \mathrm{~d}$ after transfection with control siRNA or Notch-1, -2, -3, or -4 siRNA using the Amaxa Nucleofector $\left({ }^{*} p<0.05\right.$ and $\left.{ }^{* *} p<0.01\right)$. C, 0308 glioma stem cells were transfected as in $\boldsymbol{B}$ with four different control siRNAs and siRNAs to Notch-1 through -4 , and after 3 d luciferase activity and protein concentration were quantified. siRNAs to all four Notch family members significantly decreased Notchactivity $\left({ }^{*} p<0.05\right)$.D, miR-326 causes apoptosis in GTSCs as evidenced by DNA fragmentation. Propidium iodide cell cycle assay of 0308 glioma stem cells by FACS $6 \mathrm{~d}$ after transfection with control pre-miR or pre-miR-326. E, GTSCs transfected with miR-326 undergo apoptosis demonstrated by cleaved PARP and caspase activity. Immunoblots of full-length and cleaved PARP protein in 0308 glioma stem cells $6 \mathrm{~d}$ after transfection with control pre-miR or pre-miR-326. Bottom, $\alpha$-Tubulin as loading control. $\boldsymbol{F}$, Plot of caspase-3/7 activity normalized with protein concentration in 0308 glioma stem cells $4 \mathrm{~d}$ after transfection with control pre-miR or pre-miR-326 ${ }^{*} p<$ 0.05). G, Decreased growth of GTSC lines transfected with miR-326. Shown is a plot of cell counts performed $5-6 \mathrm{~d}$ after transfection with control pre-miR or miR-326 in 0308 and 1228 glioma tumor stem cells ( ${ }^{*} p<0.05$ ). miR-326 transfection decreases invasiveness and clonogenicity of GTSC lines. $\boldsymbol{H}$, Plot showing mean number of invaded 0308 tumor stem cells $3 \mathrm{~d}$ after transfection with control pre-miR or pre-miR-326 using the modified Boyden Chamber assay coated with type IV collagen $\left({ }^{*} p<0.05\right)$. I, Plot showing the mean number of colonies formed in soft Agar by 0308 and 1228 brain tumor stem cells $3 \mathrm{~d}$ after transfection with control pre-miR or pre-miR-326 $\left.{ }^{* *} p<0.01\right)$.

down in glioma cells. Cotransfection with a miR-326 inhibitor partially but significantly protected a glioma line from Notch-1 siRNA, indicating that increased expression of miR-326 contributes to Notch-1 siRNA-induced cell death (Fig. 6B).

\section{Discussion}

Little is currently known of microRNA interactions with major cellular pathways such as Notch, for which our grasp of regulators and mediators is still murky. Previous studies have reported as- 

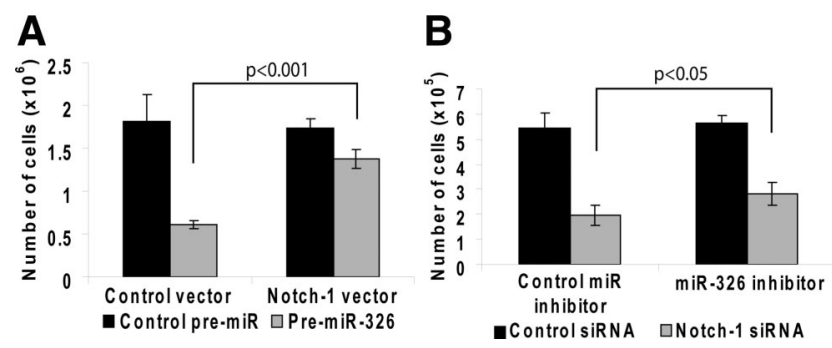

Figure 6. Notch and miR-326 effects are each partially mediated by suppression of the other. A, Restoration of Notch partially rescues from the cytotoxic effects of miR-326 transfection. Shown is a plot of cell counts of T98G glioma cells $6 \mathrm{~d}$ after transfection with control pre-miR or pre-miR-326 alone and $5 \mathrm{~d}$ together with empty plasmid vector or full-length Notch-1 plasmid. $B$, Inhibition of miR-326 partially rescues from the cytotoxic effects of efficient Notch-1 knockdown. Shown is a plot of cell counts of T98 $\mathrm{G}$ glioma cells $6 \mathrm{~d}$ after transfection with control siRNA or Notch-1 siRNA together with control miR inhibitor or miR-326 inhibitor.

sociations of a few specific microRNAs with the Notch pathway in Drosophila and Caenorhabditis elegans (Lai et al., 2005; Yoo and Greenwald, 2005). We show here that miR-326 inhibits the Notch pathway and is in turn inhibited by Notch, establishing a novel regulatory feedback loop. In addition, we demonstrate that miR-326 is downregulated in glioblastomas relative to normal brain, likely through decreased transcription of its host gene. Exogenous expression of miR-326 decreases cell viability and invasion of both established and stem cell-like glioma lines. Furthermore, transfection of glioma cells with miR-326 reduced their in vivo tumorigenicity in an orthotopic model. Importantly, upregulation of miR-326 mediates some of the cytotoxic effect of Notch inhibition in glioma cells, demonstrating biological relevance of the Notch/miR-326 relationship. This is analogous to the previously reported role of $\mathrm{miR}-34$ as a partial mediator of the pro-apoptotic effects of p53 (Chang et al., 2007; He et al., 2007; Raver-Shapira et al., 2007). In total, our findings suggest miR-326 as a potential tumor suppressor gene in glioma cells. Other work hints at dysregulation and possible roles for miR-326 in different cancers as well, such as medulloblastoma, cholangiocarcinoma, and chronic lymphocytic leukemia (Meng et al., 2006; GomezBenito et al., 2007; Ferretti et al., 2009).

We demonstrate that miR-326 downregulates both Notch-1 and Notch-2, explaining its effects on Notch activity. Notch inhibition is clearly important in the cytotoxic effects of miR-326 on glioma cells, since restoration of Notch expression partially but significantly "rescues" from the phenotypic effects of miR326. Inhibition of Notch by miR-326 may help explain its cytotoxicity to stem-like glioma cells, a highly treatment-resistant subpopulation within gliomas. Finding agents effective against these cells is a high priority for neuro-oncology and for other oncologic disciplines, and it has been postulated that inhibition of stem cell pathways such as Notch may be a viable strategy. Our results show that efficient delivery of miR-326 has therapeutic potential against both glioma stem-like cells and established glioma lines.

miR-326 no doubt has other relevant targets beside Notch. One recent report describes inhibition of Smoothened expression by miR-326 in medulloblastoma cells, leading to downregulation of the Hedgehog stem cell pathway (Ferretti et al., 2008). Combined with our findings here, this raises the appealing prospect that miR-326 can target two of the most critical stem cell pathways. However, we tested the effects of miR-326 on Hedgehog activity in a glioma line using the Gli-1 promoter reporter plasmid and did not find an effect (data not shown); this may reflect varying roles for miR-326 and these pathways in different cancer types.

miR-326 was first identified among a set of microRNAs with high expression in neurons (Kim et al., 2004). Given our findings that miR-326 suppresses Notch and the well established antineuronal effects of Notch (Nye et al., 1994), one might speculate that high miR-326 expression in neurons is another mechanism by which Notch activity is inhibited in these cells. Evolutionary pressures appear to have derived several feedback loops regulating Notch activity, such as the oscillatory loop involved in Notch and Lunatic Fringe regulation (Dale et al., 2003).

In conclusion, we describe here a novel mechanism in which Notch and miR-326 each suppress the other, suggesting a Notch/ miR-326 axis. In glioblastomas, this axis is shifted toward high Notch activity and low miR-326 activity. Reversing this axis through miR-326 delivery appears to be a potential therapy, as evidenced by its ability to decrease in vivo tumorigenicity of glioma cells.

\section{References}

Amos S, Mut M, diPierro CG, Carpenter JE, Xiao A, Kohutek ZA, Redpath GT, Zhao Y, Wang J, Shaffrey ME, Hussaini IM (2007) Protein kinase C-alpha-mediated regulation of low-density lipoprotein receptor related protein and urokinase increases astrocytoma invasion. Cancer Res 67:10241-10251.

Amsen D, Blander JM, Lee GR, Tanigaki K, Honjo T, Flavell RA (2004) Instruction of distinct CD4 T helper cell fates by different notch ligands on antigen-presenting cells. Cell 117:515-526.

Chan JA, Krichevsky AM, Kosik KS (2005) MicroRNA-21 is an antiapoptotic factor in human glioblastoma cells. Cancer Res 65:6029-6033.

Chang TC, Wentzel EA, Kent OA, Ramachandran K, Mullendore M, Lee KH, Feldmann G, Yamakuchi M, Ferlito M, Lowenstein CJ, Arking DE, Beer MA, Maitra A, Mendell JT (2007) Transactivation of miR-34a by p53 broadly influences gene expression and promotes apoptosis. Mol Cell 26:745-752.

Ciafrè SA, Galardi S, Mangiola A, Ferracin M, Liu CG, Sabatino G, Negrini M, Maira G, Croce CM, Farace MG (2005) Extensive modulation of a set of microRNAs in primary glioblastoma. Biochem Biophys Res Commun 334:1351-1358.

Croce CM, Calin GA (2005) miRNAs, cancer, and stem cell division. Cell 122:6-7.

Dale JK, Maroto M, Dequeant ML, Malapert P, McGrew M, Pourquie O (2003) Periodic notch inhibition by lunatic fringe underlies the chick segmentation clock. Nature 421:275-278.

Ferretti E, De Smaele E, Miele E, Laneve P, Po A, Pelloni M, Paganelli A, Di Marcotullio L, Caffarelli E, Screpanti I, Bozzoni I, Gulino A (2008) Concerted microRNA control of Hedgehog signalling in cerebellar neuronal progenitor and tumour cells. EMBO J 27:2616-2627.

Ferretti E, De Smaele E, Po A, Di Marcotullio L, Tosi E, Espinola MS, Di Rocco C, Riccardi R, Giangaspero F, Farcomeni A, Nofroni I, Laneve P, Gioia U, Caffarelli E, Bozzoni I, Screpanti I, Gulino A (2009) MicroRNA profiling in human medulloblastoma. Int J Cancer 124:568-577.

Gaiano N, Nye JS, Fishell G (2000) Radial glial identity is promoted by Notch1 signaling in the murine forebrain. Neuron 26:395-404.

Gomez-Benito M, Vicente C, Valganon M, Bandres E, Marcotegui N, Conchillo A, Rubio-Felix D, Calasanz MJ, Girlado P, Odero MD (2007) High resolution SNP arrays and differential miRNA expression identify novel genomic aberrations in CLL. Haematologica 92 [Suppl 2]:34. Abstract 0097.

He L, He X, Lim LP, de Stanchina E, Xuan Z, Liang Y, Xue W, Zender L, Magnus J, Ridzon D, Jackson AL, Linsley PS, Chen C, Lowe SW, Cleary MA, Hannon GJ (2007) A microRNA component of the p53 tumour suppressor network. Nature 447:1130-1134.

Henrique D, Hirsinger E, Adam J, Le Roux I, Pourquié O, Ish-Horowicz D, Lewis J (1997) Maintenance of neuroepithelial progenitor cells by DeltaNotch signalling in the embryonic chick retina. Curr Biol 7:661-670.

Hsieh JJ, Nofziger DE, Weinmaster G, Hayward SD (1997) Epstein-Barr virus immortalization: Notch2 interacts with CBF1 and blocks differentiation. J Virol 71:1938-1945.

Jiang J, Lee EJ, Gusev Y, Schmittgen TD (2005) Real-time expression profil- 
ing of microRNA precursors in human cancer cell lines. Nucleic Acids Res 33:5394-5403.

Kefas B, Godlewski J, Comeau L, Li Y, Abounader R, Hawkinson M, Lee J, Fine H, Chiocca EA, Lawler S, Purow B (2008) microRNA-7 inhibits the epidermal growth factor receptor and the Akt pathway and is downregulated in glioblastoma. Cancer Res 68:3566-3572.

Kefas BA, Heimberg H, Vaulont S, Meisse D, Hue L, Pipeleers D, Van de Casteele M (2003) AICA-riboside induces apoptosis of pancreatic beta cells through stimulation of AMP-activated protein kinase. Diabetologia 46:250-254.

Kim J, Krichevsky A, Grad Y, Hayes GD, Kosik KS, Church GM, Ruvkun G (2004) Identification of many microRNAs that copurify with polyribosomes in mammalian neurons. Proc Natl Acad Sci U S A 101:360-365.

Lai EC, Tam B, Rubin GM (2005) Pervasive regulation of Drosophila Notch target genes by GY-box-, Brd-box-, and K-box-class microRNAs. Genes Dev 19:1067-1080.

Lee J, Kotliarova S, Kotliarov Y, Li A, Su Q, Donin NM, Pastorino S, Purow BW, Christopher N, Zhang W, Park JK, Fine HA (2006) Tumor stem cells derived from glioblastomas cultured in bFGF and EGF more closely mirror the phenotype and genotype of primary tumors than do serumcultured cell lines. Cancer Cell 9:391-403.

Los M, Mozoluk M, Ferrari D, Stepczynska A, Stroh C, Renz A, Herceg Z, Wang ZQ, Schulze-Osthoff K (2002) Activation and caspase-mediated inhibition of PARP: a molecular switch between fibroblast necrosis and apoptosis in death receptor signaling. Mol Biol Cell 13:978-988.

Meng F, Henson R, Lang M, Wehbe H, Maheshwari S, Mendell JT, Jiang J, Schmittgen TD, Patel T (2006) Involvement of human micro-RNA in growth and response to chemotherapy in human cholangiocarcinoma cell lines. Gastroenterology 130:2113-2129.

Morrison SJ, Perez SE, Qiao Z, Verdi JM, Hicks C, Weinmaster G, Anderson DJ (2000) Transient Notch activation initiates an irreversible switch from neurogenesis to gliogenesis by neural crest stem cells. Cell 101:499-510.

Murchison EP, Hannon GJ (2004) miRNAs on the move: miRNA biogenesis and the RNAi machinery. Curr Opin Cell Biol 16:223-229.

Nye JS, Kopan R, Axel R (1994) An activated Notch suppresses neurogenesis and myogenesis but not gliogenesis in mammalian cells. Development 120:2421-2430.

Oishi K, Kamakura S, Isazawa Y, Yoshimatsu T, Kuida K, Nakafuku M, Masuyama N, Gotoh Y (2004) Notch promotes survival of neural precursor cells via mechanisms distinct from those regulating neurogenesis. Dev Biol 276:172-184.

Purow BW, Haque RM, Noel MW, Su Q, Burdick MJ, Lee J, Sundaresan T, Pastorino S, Park JK, Mikolaenko I, Maric D, Eberhart CG, Fine HA (2005) Expression of Notch-1 and its ligands, Delta-like-1 and Jagged-1, is critical for glioma cell survival and proliferation. Cancer Res 65:2353-2363.

Raver-Shapira N, Marciano E, Meiri E, Spector Y, Rosenfeld N, Moskovits N, Bentwich Z, Oren M (2007) Transcriptional activation of miR-34a contributes to p53-mediated apoptosis. Mol Cell 26:731-743.

Stylianou S, Clarke RB, Brennan K (2006) Aberrant activation of notch signaling in human breast cancer. Cancer Res 66:1517-1525.

Thatcher EJ, Flynt AS, Li N, Patton JR, Patton JG (2007) MiRNA expression analysis during normal zebrafish development and following inhibition of the Hedgehog and Notch signaling pathways. Dev Dyn 236:2172-2180.

Weng AP, Ferrando AA, Lee W, Morris JP 4th, Silverman LB, SanchezIrizarry C, Blacklow SC, Look AT, Aster JC (2004) Activating mutations of NOTCH1 in human T cell acute lymphoblastic leukemia. Science 306:269-271.

Yekta S, Shih IH, Bartel DP (2004) MicroRNA-directed cleavage of HOXB8 mRNA. Science 304:594-596.

Yoo AS, Greenwald I (2005) LIN-12/Notch activation leads to microRNAmediated down-regulation of Vav in C. elegans. Science 310:1330-1333. 